\title{
Special Issue on Information and Communication Technologies for Smart City
}

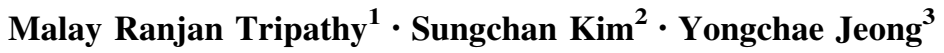

Published online: 30 July 2020

(C) The Society for Reliability Engineering, Quality and Operations Management (SREQOM), India and The Division of Operation and Maintenance, Lulea University of Technology, Sweden 2020

Rapid growth of information and communication technology (ICT) has created lots of demand to have high capacity data driven complex network, data sharing, handling, analysis, privacy and security for different applications. The convergence of information technologies with different advanced technologies such as signal and image processing, web and database technology, health care, robotics, transportation, IoT, virtual reality, embedded systems, wireless technology, bio inspired intelligence, environment, industry, communication and cultural innovation has opened up various possibilities of applications. ICT is one of the important infrastructures for reliable and sustainable smart city. It provides services which helps the efficient use of education, health care, e-governance, mass transit, security, environmental monitoring systems. It acts as a platform to aggregate information and data to support for overall understanding of city resource consumption, services and lifestyle. It enables information and knowledge sharing, forecasting, data prediction, analytics, big data, internet of things (IoT) and ubiquitous sensor networks to improve the comfort and quality of citizen's life. Therefore, this area of research is very important and interesting for academicians, scientist and team members of Industry.

Malay Ranjan Tripathy

mrtripathy@amity.edu

1 Department of Electronics and Communication Engineering, Amity University Uttar Pradesh, Noida, Uttar Pradesh, India

2 Division of Computer Science and Engineering, Jeonbuk National University, Jeonju, Republic of Korea

3 Division of Electronic Engineering, Jeonbuk National University, Jeonju, Republic of Korea
The present issue is based on the theme of IT convergence for smart society. The papers are short listed from presented papers in an International Symposium on Information Technology Convergence 2018 (ISITC 2018) which was held during October 24-27, 2018 at Jeonju, Korea.

In this special issue fourteen papers are short listed for the publication. First paper is based on Clustering-Based Privacy Preserving Anonymity Approach for Table Data Sharing. It is a challenging task to give guarantee of citizen's privacy while sharing government data for the improvement of government governance and service levels. In general available government data are diversity types and complex in nature. Cluster-based anonymous table data sharing privacy protection method (CATDS) is proposed in this paper. It is compared with the classical k-anonymity algorithm which is known as Incognito algorithm. It is proved that CATDS algorithm can effectively reduce the amount of information loss and improve the availability of shared table data while protecting the private information of shared table data.

The second paper is based on the complexity reduced soft MIMO detection using single tree search. The soft iterative detection and decoding can produce the near capacity performance in MIMO system. The single tree search (STS) which is based on sphere decoding can produce near optimal performance in iterative detection and decoding. In general it is computationally complex and complexity increases with the increase in the iterations. In this paper a modified STS method is proposed. It is shown to have reduced computational complexity with negligible performance degradation compared to the conventional full search and STS methods. 
The third paper is based on the analysis and reflection on peer assessment results based on short play of Game Theory. Peer assessment is an important part to evaluate college students' learning effect and ability in blended learning. However, less effort has been made to understand the challenges to achieve the quality of peer assessment. By Game Principles Course, this research puts forward the idea of building a peer assessment loop to improve the quality of peer assessment and prevent the bad results of peer assessment.

The fourth paper is based on marker selection for predicting continuous survival period of colorectal cancer. The colorectal cancer is one of the most prevalent cancers that usually has a stronger concealment. For early screening or preventing colon cancer, various type of biomarkers are checked to verify whether they can accurately and sensitively assess this disease. However, in this paper, a hybrid algorithm (BPPSO) based on particle swarm optimization combining with BP neural network is proposed to select critical biomarkers for predicting continuous survival period. The experiments show that BPPSO is effective in biomarker selection.

The fifth paper is based on the design and implementation of tunnel image mosaic system based on Open CV. With the development of transportation, more and more tunnels used to have appeared. It is becoming very difficult to monitor the complete tunnel situation. This leads to increase in the vehicle traffic risk index in the tunnel. For this, the tunnel mosaic technology based on ORB algorithm is proposed in this paper to improve the monitoring of tunnel environment.

The sixth paper is based on the resource oriented topology construction to ensure high reliability in IoT based smart city networks. To improve the network reliability efficient parent selection scheme is proposed to avoid the overburdened parent. The scheme combines the multiple routing metrics to calculate the rank and that result in the balanced topology formation which ensures the higher reliability in routing protocol for low power and lossy network (RPL) based advanced metering infrastructure (AMI) network.

The seventh paper is based on the research on power consumption information acquisition system based on high speed carrier interconnection protocol. This is an interesting work. The communication performance with networking and business support capabilities is verified and evaluated.

The eighth paper is based on the depth edge detection using edge-preserving filter and morphological operations. In this paper Canny edge detection principle and morphological operations have been used for depth edge detection. It is shown that this method can detect edges of a depth image better than the method without the edge-preserving filter such as gaussian and median blur.

The ninth paper is based on the deep feature representation and Ball-tree for face sketch recognition. Some of interesting results are presented in this paper. The experimental results on CUFS and IIIT-D datasets demonstrate the superiority of the proposed method compared with existing algorithms.

The tenth paper is based on energy load forecasting (ELF) model based on deep neural networks for smart grids. This model is used to manage the energy consumption in smart grids. Two different types of deep neural network architectures such as deep feedforward neural network (Deep-FNN) and deep recurrent neural network (Deep-RNN) are studied. The simulation results are compared in terms of mean absolute percentage error (MAPE). The results show that the proposed ELF model has attained better generalization and outperform the existing load forecasting models based on the shallow neural network (SNN), ensemble tree bagger (ETB) and generalized linear regression (GLR).

The eleventh paper is based on the short-term multiple power type prediction based on deep learning. It is an interesting paper. A model on four layer of deep neural network (DNN) model by stacked Denoising Auto-encoders (SDAE) to analyse four types of power data: current $(\mathrm{I})$, voltage $(\mathrm{U})$, active power $(\mathrm{P})$ and Reactive power $(\mathrm{Q})$ is proposed. It is found that the 4-layer network is better than that of the 3-layer, 5-layer and 7-layer network models.

The twelfths paper is based on a signal screening based sequence-correlation localization algorithm for wireless sensor networks. The objective of this work is to design a Received Signal Strength (RSS) based range-free localization technique called signal screening based sequencecorrelation localization algorithm (SCLSS) which can provide accurate and cost efficient localization performance for WSNs. Detail discussion is made on this model. The SCLSS uses the weighted centroid approach to isolate the location where the unknown node most probably resides. Simulation results show that the proposed SCLSS offers more accurate localization with fewer anchor nodes compared with its counterparts.

The thirteenth paper is based on the multi-focus image fusion via NSST with non-fixed base dictionary learning. The multi-focus image fusion via non-subsampled shearlet transform (NSST) with non-fixed base dictionary learning is discussed in this paper. First, low frequency coefficients and high frequency coefficients are obtained by NSST. Then, a new strategy, which can enhance the information of spatial detail for the fused image is proposed to process two different coefficients. The low frequency coefficients are fused via a non-fixed base dictionary, which makes the K-SVD algorithm more efficient, and the high frequency 
coefficients are fused with spatial frequency (SF), which is effective in the fused image. Experiment results demonstrate that the results of proposed method obtain more spatial details and have almost zero residuals compared with several conventional methods in terms of both visual quality and objective measurements.

The fourteenth paper is based on the parallel voltage sag compensator without an injection transformer. The proposed PVSC has a bidirectional DC-DC converter to transfer the electrical power between the energy storage device and the bidirectional inverter. It uses the commonmode filter capacitors for the AC-side filter in the bidirectional inverter. The ground leakage current can be effectively suppressed between the energy storage device and the AC voltage. The proposed system has high power efficiency and low ground leakage current. Experimental results are discussed to evaluate the performance of the proposed system.

In conclusion to this introduction to the special issue on Information and Communication Technologies for Smart City, we believe that readers will find it useful for their future reference. Most of the papers are interesting and addressed different use cases relevant to smart city applications. We thank to the Editor in chief, Springer Staffs and reviewers involved in this special issue of International Journal of System Assurance Engineering and Management for their help and support. 